\title{
Dopamine Receptor-Specific Contributions to the Computation of Value
}

\author{
Christopher J Burke,,I, Alexander Soutschek', Susanna Weber', Anjali Raja Beharelle', Ernst Fehr', \\ Helene Haker ${ }^{2}$ and Philippe N Tobler' \\ 'Laboratory for Social and Neural Systems Research, Department of Economics, University of Zurich, Zurich, Switzerland; ${ }^{2}$ Translational \\ Neuromodeling Unit, Institute for Biomedical Engineering, ETH Zurich, Zurich, Switzerland
}

\begin{abstract}
Dopamine is thought to play a crucial role in value-based decision making. However, the specific contributions of different dopamine receptor subtypes to the computation of subjective value remain unknown. Here we demonstrate how the balance between DI and D2 dopamine receptor subtypes shapes subjective value computation during risky decision making. We administered the D2 receptor antagonist amisulpride or placebo before participants made choices between risky options. Compared with placebo, D2 receptor blockade resulted in more frequent choice of higher risk and higher expected value options. Using a novel model fitting procedure, we concurrently estimated the three parameters that define individual risk attitude according to an influential theoretical account of risky decision making (prospect theory). This analysis revealed that the observed reduction in risk aversion under amisulpride was driven by increased sensitivity to reward magnitude and decreased distortion of outcome probability, resulting in more linear value coding. Our data suggest that different components that govern individual risk attitude are under dopaminergic control, such that D2 receptor blockade facilitates risk taking and expected value processing.

Neuropsychopharmacology (2018) 43, I4I5-1424; doi:10.1038/npp.2017.302; published online 31 January 2018
\end{abstract}

\section{INTRODUCTION}

Risk is common in our lives and affects many everyday decisions (eg, whether to gamble in the casino, which insurance policy to purchase, or which school to enroll in). When making decisions between risky options, people need to balance the magnitudes of potential gains and losses with the probabilities that they will occur. One possibility is to multiply the magnitudes of risky outcomes by their respective probabilities to calculate each choice option's expected value and choose the option with higher expected value irrespective of risk (Pascal, 1948). However, behavioral evidence indicates that people have individually different risk attitudes, and therefore value risky options differently. This often results in options with lower expected value being chosen if the alternative option has higher risk (Christopoulos et al, 2009), a phenomenon known as risk aversion.

A highly influential psychological model to describe this behavior, prospect theory (Kahneman and Tversky, 1979), postulates that risk-sensitive economic choice can be described by nonlinear conversion of objective outcome magnitudes and probabilities into subjective value, or utility.

\footnotetext{
*Correspondence: Dr CJ Burke, Department of Economics, University of Zurich, Blumlisalpstrasse 10, CH-8006 Zurich, Switzerland, Tel: +4 I 4463448 38, E-mail: cjohnburke@gmail.com

Received 21 July 2017; revised 7 November 2017; accepted 8 December 2017; accepted article preview online 18 December 2017
}

Specifically, prospect theory postulates that preferences over risky options can be explained by three parameters that define a person's utility function. First, the curvature of the utility function, $\sigma$, captures whether an individual is risk averse (concave utility function) or risk seeking (convex utility function). A second parameter, $\alpha$, governs how much probabilities are distorted, with smaller probabilities typically being overweighted and larger probabilities underweighted. Third, a parameter $(\lambda)$ that controls how much steeper the utility function is for losses than for gains captures the degree of loss aversion. Together, these parameters describe utility functions that model subjective risk attitudes by governing how individuals respond to the magnitudes and probabilities of outcomes encountered in risky options. Prospect theory was introduced because it can explain behaviors that more traditional models such as expected utility theory (Bernoulli, 1954; Von Neumann and Morgenstern, 2007) cannot explain. According to expected utility, risk preferences are driven by the curvature of the utility function, without probability distortion or loss aversion.

The neural basis for value encoding under risk has been widely investigated, with the dopaminergic system emerging as a neural substrate for processing economic value. Dopamine neurons encode reward magnitude (Schultz, 1998) and combine reward magnitude with probability (Tobler et al, 2005) into prospect theory- or expected utility-like value signal of gains in both risky and safe options (Stauffer et al, 2014). In addition, people suffering from disease- or drug-induced modifications to the dopaminergic 

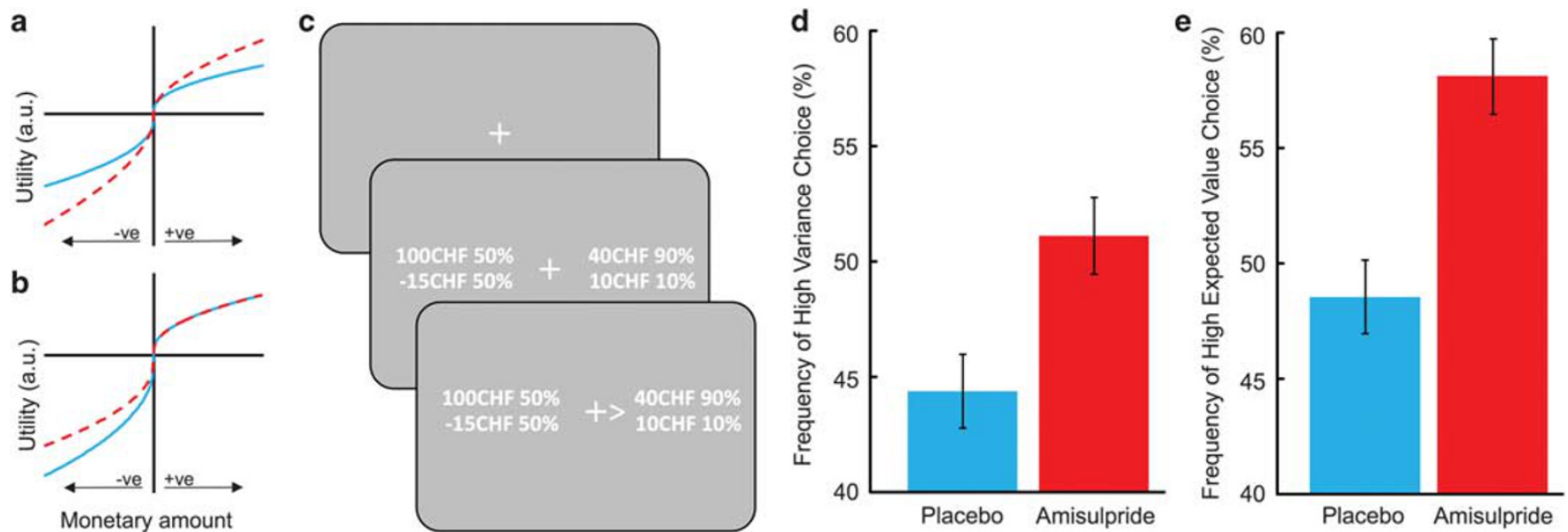

Figure I Potential effects of amisulpride on utility function, illustration of example trial, and observed effects of amisulpride on choice behavior. (a, b) Prospect theory proposes an asymmetrical, nonlinear, and concave and convex mapping of subjective value on increasing monetary gains and losses that results in different risk attitudes of individuals. (a) Potential effect of D2/D3 blockade on reducing the curvature of the value function (red dashed line; prospect theory utility curvature parameter $\sigma=0.7$ ) compared with placebo (blue; $\sigma=0.5$ ). (b) Potential effect of D2/D3 blockade on reducing loss aversion (red dashed line; prospect theory loss aversion parameter $\lambda=1.5$ ) compared with placebo (blue line; $\lambda=2$ ). Note that solely manipulating the loss aversion parameter does not affect value sensitivity in the gain domain. (c) Example trial. After a fixed intertrial interval of $2 \mathrm{~s}$, participants made a self-paced choice (20 in total) between risky options that varied in gain and loss magnitudes and probabilities and that were presented on the left and right sides of the screen. (d) Participants in the amisulpride group chose the riskier (higher variance) option significantly more often than participants in the placebo group, indicating decreased risk aversion. (e) Amisulpride resulted in more frequent choices of the high expected value option, consistent with increased value sensitivity.

system demonstrate differences in risky decision making (Rogers et al, 1999; Bornovalova et al, 2005; Leland and Paulus, 2005). Rodent studies have shown that reward value (Howe et al, 2013) and the subjective value of risk (Sugam et al, 2012) is expressed in dopamine release. Finally, risky decision making can be modulated by stimulating the inputs to dopamine neurons (Stopper and Floresco, 2014), dopamine neurons themselves (Stopper et al, 2014), or striatal D2 receptor neurons (Zalocusky et al, 2016), and receptorspecific dopaminergic drugs can bias preferences for risky $v s$ safe outcomes (Stopper et al, 2013). However, the question of precisely how the dopaminergic system processes the components of subjective risk attitude and how the different parameters governing it are mediated by different dopamine receptor subtypes remains unanswered.

One recent model has proposed that risk attitude is governed by the balance of D1- and D2-receptor-mediated activity in the dopaminergic system (Clark and Dagher, 2014; Mikhael and Bogacz, 2016). The model is compatible with animal literature (Kravitz et al, 2012; Lee et al, 2016; Surmeier et al, 2014; Tai et al, 2012) but remained largely untested. Specifically, it proposes that the subjective sensitivity to potential rewards in risky options (ie, utility curvature) is D1 mediated, and the subjective sensitivity to potential punishments (ie, loss aversion) is D2 mediated. Without pharmacological intervention, reward sensitivity is typically reduced for larger rewards, reflected in concave utility functions from which risk aversion partly arises. By extension, a reduction in D2-mediated (inhibitory) activity should enhance sensitivity to large monetary rewards in risky options, resulting in more linear (or possibly convex) utility functions and reduced risk aversion.

To test this idea, we pharmacologically blocked D2 receptors while participants made choices between risky options. We hypothesized that D2 antagonism would decrease risk aversion by increasing the sensitivity to larger reward magnitudes and thereby reducing the concavity of the utility function for gains (Figure 1a). In addition, risk aversion could also be modulated through a reduction in probability distortion, allowing people to more accurately compute the probabilities associated with risky outcomes, or through a decrease in loss aversion (Figure 1b), making people less sensitive to potential negative outcomes from risky options. Any of these effects would predict that an expected utility model would fit the data better than a prospect theory model under D2 antagonism. To test these different possibilities, we conducted a randomized doubleblind, placebo-controlled pharmacological intervention in two groups of healthy participants while they performed a dynamic risky decision-making task (Figure 1c) designed to elicit their underlying risk preferences.

\section{MATERIALS AND METHODS}

\section{Participants}

Using a randomized double-blind, placebo-controlled, between-subjects design, we recruited 93 participants from the student population at the University of Zurich and randomly assigned them to one of the two groups. Both groups were matched in terms of baseline measures that may influence drug effects on the dopaminergic system, including age, sex, BMI, working memory capacity (digit span task), and mood, alertness, and calmness, that were measured using the multidimensional mood state (MDMQ) questionnaire (Steyer et al, 1997). One group received placebo $(n=48)$, whereas the other received a $400 \mathrm{mg}$ dose of amisulpride $(n=45)$. The results reported here form part of a larger study. In addition to the risky decision task, participants performed temporal and effort discounting tasks, a prosocial decision-making task (Soutschek et al, 2017), a reversal learning task, and a stop-signal-reaction time task. 


\section{Pharmacological Manipulation}

Amisulpride is a dopamine antagonist that selectively blocks neurotransmission at dopamine D2/D3 receptors (Rosenzweig et al, 2002). At low doses (50-300 mg), amisulpride primarily prevents dopamine action at presynaptic D2/D3 autoreceptors (Racagni et al, 2004), thus effectively stimulating dopaminergic neurotransmission. At higher doses $(\geqslant 400 \mathrm{mg})$, amisulpride preferentially antagonizes postsynaptic D2/D3 receptors (Schoemaker et al, 1997). The used dose of $400 \mathrm{mg}$ is thus at the lower end of postsynaptically active high doses (Müller et al, 2002; Rosenzweig et al, 2002) and occupies $~ 70 \%$ of D2 receptors (Meisenzahl et al, 2008). To limit extrapyramidal side effects, we refrained from using higher doses. Two plasma concentration peaks are typically observed during the absorption period, with the first, lower peak occurring $\sim 1-$ $2 \mathrm{~h}$ after ingestion (Le Bricon et al, 1996).

\section{Task}

After receiving placebo or amisulpride, participants waited for $1.5 \mathrm{~h}$ in the controlled laboratory environment. Participants then performed a risky decision-making task (Figure 1c) that consisted of 20 trials where they made choices between two risky options presented at the same time on the screen. The task was programmed using the Cogent toolbox (v1.32) and MATLAB (R2016b). All decisions were between two compound lotteries of the form $p$ chance of magnitude $x, 1-p$ chance of magnitude $y$. Lotteries were constructed on a trial-bytrial basis by combining different levels of $\{x, p, y\}$, where $p \in\{0.1,0.3,0.5,0.7,0.9\}, \quad x \in\{1,30,40,100,1000\}, \quad$ and $y \in\{-20,-15,-10,-5,5,10,30\}$, with $x$ and $y$ denominated in Swiss francs (CHF). On every trial, one lottery was presented on the left side of the screen and one on the right side, with the magnitudes and their associated probabilities on the same horizontal plane. For example, Figure 1c illustrates a choice between a lottery on the left side that results in a gain of 100 Swiss francs with $50 \%$ chance or a loss of - 15 Swiss francs with $50 \%$ chance and a lottery on the right side that results in a gain of 40 Swiss francs with $90 \%$ chance or 10 Swiss francs with $10 \%$ chance. To ensure incentive compatibility, one trial was randomly selected at the end of the experiment and the lottery chosen by the participant in that trial was realized. The outcome was added to or subtracted from the fee participants received for taking part (120 Swiss francs) in the pharmacological experiment. Specifically, participants were instructed to treat every decision as if it were the one being selected at the end and therefore make their choices according to their true preferences. Average payout was 22.3 Swiss francs; 28 participants incurred losses.

\section{Dynamic Task Design}

After each choice, the task adaptively presented to the participant a new pair of lotteries that optimized the sequence of possible trials to recover the participant's true risk preferences. In such a way, each new lottery pair maximized the amount of information about the participant's risk attitude, given their decisions on preceding trials. We implemented the adaptive Bayesian method described by Toubia et al (2013), where the posterior distribution over prospect theory parameters is updated after each choice and the task selects a new pair of lotteries that maximizes the amount of information over the parameters to home in on the participant's true risk attitude (Supplementary Material; Dynamic Task Design). This Bayesian approach to adaptive elicitation of risk attitude differs from the typical bisection approaches used in psychophysics (Cornsweet, 1962) and allows accurate elicitation of risk preferences within 20 trials (Supplementary Figure S1) by adapting both the probabilities and magnitudes for both options on every trial (as opposed to keeping one option fixed as in more traditional staircase/ bisection approaches).

\section{Simulations}

Simulations confirmed that the method could recover true parameter values within 20 trials (Supplementary Figure S1) and was robust to different priors (Supplementary Figure S2). Simulations were also conducted to assess the unique impact of each parameter on choices (Supplementary Figure S3). Full details of these simulations can be found in the Supplementary Material.

\section{Data Analysis}

Choice frequency data and response times were analyzed using the statistics toolbox of MATLAB in a series of $t$-tests and ANOVAs. The specific behavioral measures used in our model-free analysis were the proportions with which (1) the higher variance or (2) the higher expected value lottery was chosen. Mixed effects multiple and logistic regressions were carried out in R. For the expected value sensitivity analysis (Figure 2d), we computed the expected values of both options for each trial, binning these values around 10, 20, 50, $70,100,500$, and $1000 \mathrm{CHF}$ and created dummy variables to encode the presence of each expected value in the high-risk $(+1)$ or low-risk $(-1)$ options on each trial, and regressed participants' choices for the high-risk option against these dummy variables using a mixed effects logistic regression that included both fixed and random effects for each participant. Regression parameters for each dummy variable (ie, the influence of each expected value on risky choices) were normalized between 0 and 1 , directly compared using ANOVA, and plotted in Figure 2d.

\section{Choice Models and Fitting}

Prospect theory model fitting (Figures 2 and 3) was applied in MATLAB according to the hierarchical Bayesian framework described in detail in Toubia et al (2013) using the standard cumulative prospect theory model used in the literature (Tversky and Kahneman, 1992) with standard probability weighting (Prelec, 1998) to the placebo and amisulpride groups separately. Our use of group-wise hierarchical Bayes was motivated by two factors: (1) the relatively large number of participants in each group and (2) relatively small number of trials per participant. Options are defined by $\{x, p ; y\}$ with outcome $x$ occurring with probability $p$ and outcome $y$ occurring with probability $1-p$. Formally, the value of an option to a participant is given by:

$U(x, p, y, \alpha, \sigma, \lambda)= \begin{cases}v(y, \sigma)+\pi(p, \alpha)(v(x, \sigma)-v(y, \sigma)) & \text { if } x>y>0 \text { or } x<y<0 \\ \pi(p, \alpha) v(x, \sigma)+\pi(1-p, \alpha) v(y, \sigma) & \text { if } x<0<y\end{cases}$

(1) 


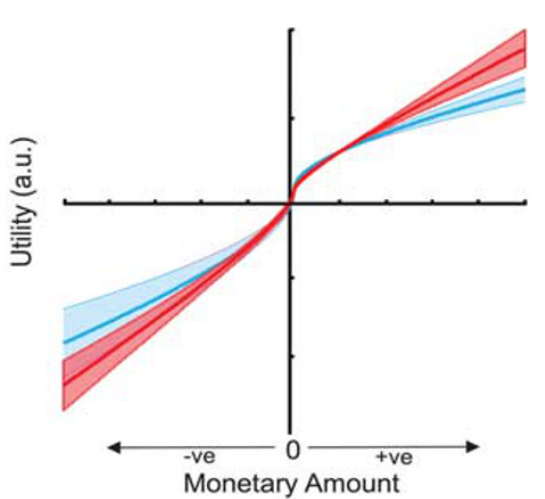

c

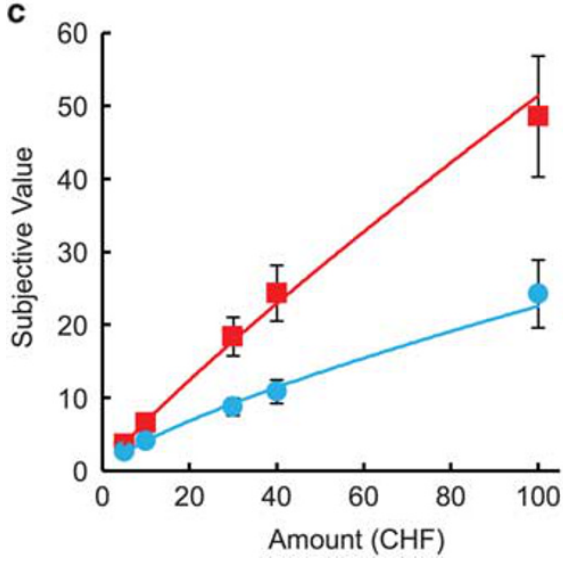

b

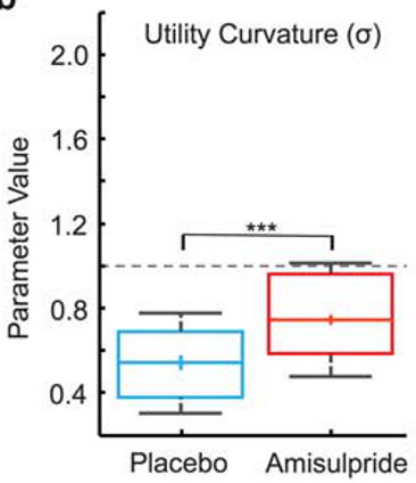

d

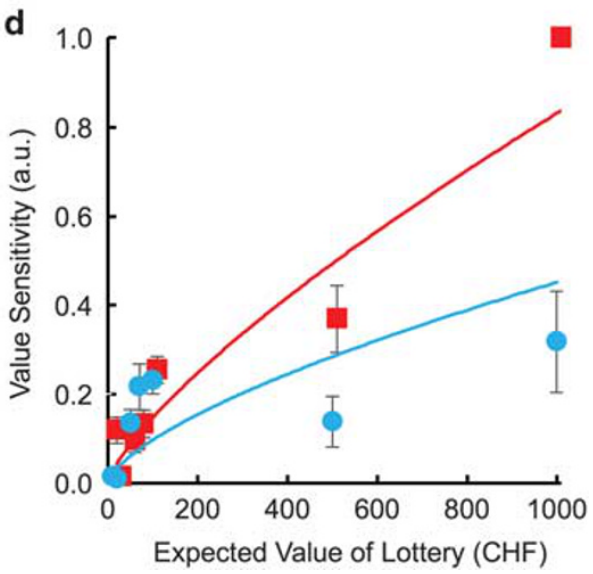

Figure 2 Observed effects of amisulpride on subjective value function from prospect theory. (a) Fitting a prospect theory model to participants' choices revealed a significantly more linear value function for the amisulpride (red) relative to the placebo group (blue). (b) These findings were supported by utility curvature $(\sigma)$ parameters being significantly closer to I, ie, linearity (gray dashed line) in the amisulpride group. Boxplots show that the mean $(+)$ and median ( - ) coincided; **** indicates $p<0.00 \mathrm{I}$; boxes represent 25 th and 75 th percentiles and whiskers the 9th and the 9 I st percentile. (c) Recovered value functions for different reward magnitudes revealed a steeper and more linear function for the amisulpride group (red), indicating lower risk aversion when compared with the placebo group (blue) (d). As both utility curvature and probability weighting (Figure 3) functions were more linear under amisulpride, sensitivity to increases in the expected value of the option should also be increased. This was confirmed by a logistic regression of choices of the higher expected value option against the expected value of the option that allowed us to estimate regression weights as a proxy for the expected value sensitivity in the two groups. Participants rarely encountered extremely high option magnitudes as they were associated with high risk. Accordingly, we could not plot an error bar for the amisulpride group at CHF 1000. Error bars represent the SEM.

where

$v(x, \sigma)= \begin{cases}x^{\sigma} & \text { for } x>0 \\ -\lambda\left(-x^{\sigma}\right. & \text { for } x<0\end{cases}$

and

$\pi(p, \alpha)=\exp \left[-(-\ln p)^{\alpha}\right]$

In the hierarchical Bayesian framework, a uniform prior distribution of the model parameters is specified and a posterior distribution is calculated by obtaining the prior with the choice data from the participants. The hierarchical Bayesian approach also allows more accurate group estimates by simultaneously leveraging individual- and group-level estimates (utility curvature, $\sigma$; probability distortion, $\alpha$; and loss aversion, $\lambda$ ), such that individual estimates deemed to be unlikely, ie, outliers given the group distribution, receive less weight than more reliable measures. The uniform prior ensures that the mode of the posterior distribution (maximum a posteriori) is equivalent to the maximum likelihood estimate (Toubia et al, 2013; p 620).
We assessed the fit of each model for each participant based on the softmax choice rule (Supplementary Methods). Expected utility and expected value models were fitted using maximum likelihood estimation. Formally, the expected utility of an option is given by:

$U(x, p, y, \sigma)=\pi(p) v(x, \sigma)+\pi(1-p) v(y, \sigma)$

where

$v(x, \sigma)=x^{\sigma}$

and the expected value of an option is given by:

$U(x, p, y)=\pi(p)(x)+\pi(1-p)(y)$

\section{RESULTS}

In line with the observation that humans typically are risk averse (see, eg, Christopoulos et al, 2009), analysis of the choice data revealed that the placebo group was risk averse, with the proportion (45.2\%) of choices of the riskier option being significantly smaller than $50 \%$ (one-sample $t$-test, $t_{47}=-2.78, p=0.008$ ), even though the expected value of the 
a
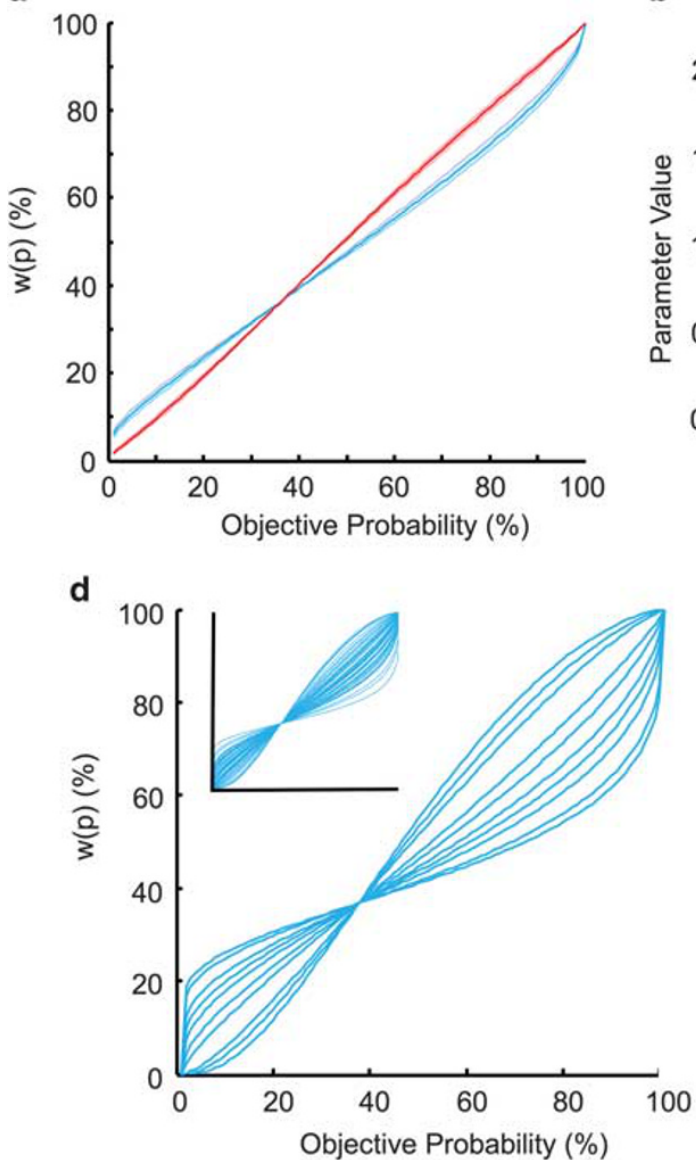

b

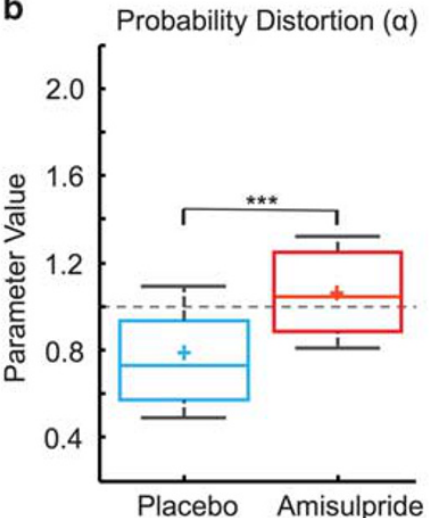

C

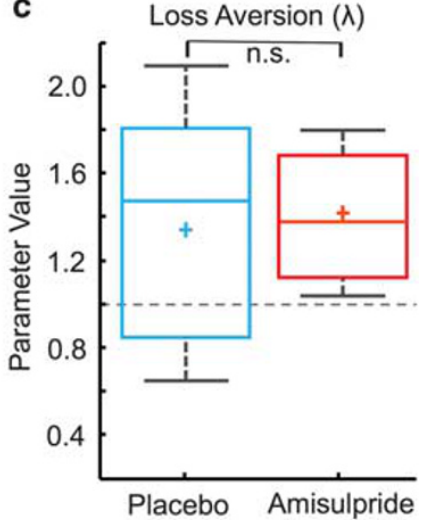

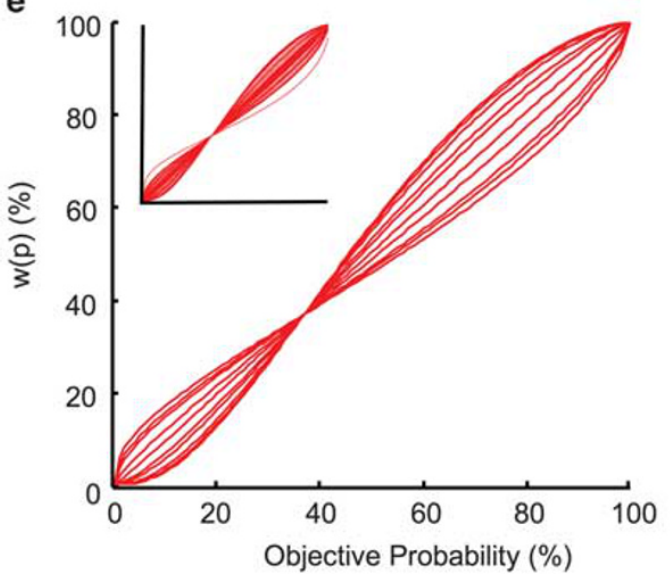

Figure 3 Effects of amisulpride on probability distortion and loss aversion. (a) Average probability weighting functions were less distorted in the amisulpride group (red) than in the placebo group (blue). (b) Accordingly, probability distortion ( $\alpha$ ) parameters were significantly closer to I for the amisulpride compared with the placebo group. (c) Groups did not differ significantly in mean loss aversion ( $\lambda$ ) parameters. In (b) and (c), boxplots show the mean ( + ) and median $(-)$; **** indicates $p<0.00$ I; n.s. indicates no significant difference; boxes represent 25 th and 75 th percentiles and whiskers the 9 th and the 91 st percentile, with the gray dashed line representing I (ie, linearity). (d) To better visualize individual probability weighting functions we randomly selected I 5 participants in the placebo group (whole group shown in inset). Similar to the full group, probability distortions were more pronounced and varied more widely in these participants. (e) In contrast, the amisulpride group showed more homogeneous and reduced probability distortions, both in randomly selected I 5 participants and in the full group (inset).

safer options was smaller than that of the riskier options. Importantly, compared with the placebo group, participants under dopamine D2/D3 receptor blockade were significantly less risk averse, showing more choices of the higher risk option (mixed effects logistic regression with risky choice as dependent variable, treatment as primary independent variable, and participant as a random independent variable, $\chi^{2}=5.14, p=0.023$; Figure 1d). This effect also arose when we entered working memory capacity (a proxy for baseline dopamine synthesis capacity) as a regressor of no interest into the model $\left(\chi^{2}=5.01, p=0.025\right)$. Please note that amisulpride did not cause participants to become risk seeking, as the proportion (50.2\%) of high variance choices in the treatment group was not significantly different from $50 \%$ (one-sample, one-tailed $t$-test, $t_{44}=0.49, p=0.62$ ), suggesting that under amisulpride participants were risk neutral.

Participants under amisulpride were also more likely to choose the higher expected value option than participants under placebo (mixed effects logistic regression, $\chi^{2}=6.89$, $p=0.008$; with working memory capacity as a regressor of no interest: $\chi^{2}=6.46$ and $p=0.011$; Figure 1e). Although risk and expected value are necessarily correlated in our task (to avoid decisions where one option strictly dominates the other), we ran a multiple mixed effects logistic regression to assess if amisulpride affected high expected value choices and higher risk choices to different degrees. There was no significant difference in the regression weights between expected value and risk (one-sample $t$-test, $t_{147}=-1.11$; $p=0.85$ ), suggesting that reduced risk aversion under amisulpride arose from similar effects on expected value and risk processing.

To determine whether reduced risk aversion in the amisulpride group could be explained by increased choice randomness under amisulpride, we ran a test assessing the number of consecutive choices above or below the mean of the choice vector (implemented using Matlab's 'runstest' function). This showed that the choice profile in both the amisulpride and placebo groups was not random $(Z=-1.09$; $p=0.28$ ). Additionally, participants under amisulpride were less random in their choice of the higher expected value option than participants under placebo (Figure 1e). Moreover, model-based analyses showed that if anything, amisulpride was associated with less variation in behavior, 
both in probability distortion and loss aversion (see below). Finally, response times did not differ between the amisulpride and placebo groups (two-sample $t$-test, $t_{91}=-0.44$; $p=0.66$ ), providing no evidence that the amisulpride group made choices less carefully than the placebo group.

A decrease in risky choices under amisulpride could arise from increased linearity in the curvature of the utility function, a reduction in loss aversion, changes in the probability weighting function, or a combination. To investigate these possible channels of amisulpride action, we recovered the $\sigma, \lambda$, and $\alpha$ parameter values for each participant by fitting a prospect theory model to the choice data using hierarchical Bayes. Using the average parameter values from the fitting, we plotted the recovered utility function across arbitrary amounts. Visual inspection of this average utility function (Figure 2a) showed a more linear function (and decreased probability distortion; see below and Figure 3) for the amisulpride group. This impression was confirmed by directly comparing the best fitting parameters, with the amisulpride group showing significantly less curvature (ie, the $\sigma$ parameter was closer to 1 ) than the placebo group (amisulpride $=0.741 ;$ placebo $=0.548 ; t_{91}=$ - 3.82; $p=0.0001$; two-sample $t$-test; $p=0.0003$ when controlling for working memory; $p=0.0002$ when controlling for weight; Figure 2b). Thus, participants under amisulpride exhibited significantly decreased concavity in their utility functions.

To further illustrate these findings, we next used the individual utility curvature parameters to transform objective outcome magnitudes into subjective values. Using the best fitting utility curvature parameter $(\sigma)$ for each participant, we inferred the subjective value of objective reward magnitudes with the power utility function specified according to prospect theory (ie, objective monetary magnitude in Swiss francs raised to the power of $\sigma$ for each participant). A twoway ANOVA with objective monetary magnitude (CHF 5, $10,30,40$, and 100) and treatment (amisulpride/placebo) as factors revealed a significant interaction effect of treatment and monetary magnitude $\left(\mathrm{F}_{4,92}=4.07, p=0.003\right)$, with participants under amisulpride showing increased sensitivity to outcome magnitudes as these magnitudes increased (Figure 2c). Thus, behavioral choices under amisulpride were in agreement with the notion that participants assessed gain and loss magnitudes in the risky options in a more linear and objective manner.

Next, we assessed whether the more linear value function under amisulpride (Figure 2c) translated into increased sensitivity to expected value. To test this possibility, we calculated the expected values (probability $\times$ magnitude) of all options presented to each participant. Then, we performed a mixed effects logistic regression to assess the influence of expected value on the probability of participants selecting the higher expected value option. If amisulpride increased expected value sensitivity, one would expect to see significantly larger regression weights for high expected values in the amisulpride group. Indeed, a two-way ANOVA revealed a significant interaction effect of high (larger than median rank of 100 Swiss francs) vs low (smaller than median) expected values with placebo $v s$ amisulpride treatment $\left(\mathrm{F}_{2,10}=4.99 ; p=0.046\right)$, indicating higher expected values had a significantly different impact on choices for the two groups (Figure 2d). Fisher's $r$-to- $z$ transformation confirmed that the increasing value sensitivity was significantly more tightly correlated to increasing expected value in the amisulpride group compared with the placebo group $(Z=2.1, \quad p=0.02)$. These data suggest that the value processing system under amisulpride indeed becomes more sensitive to increases in expected value.

Because expected value incorporates both magnitude and probability information, one possibility is that amisulpride affects not only value curvature but also probability distortion. Indeed, visual inspection of the probability weighting function for the two groups suggests that amisulpride reduced probability distortion (Figure 3a). To test this possibility quantitatively, we directly compared the probability distortion parameters for the two groups. Compared with placebo, probability distortion was significantly lower under amisulpride (amisulpride $=1.059$; placebo $=0.804 ; \quad t_{91}=-4.63 ; \quad p=0.0001 ;$ two-sample $t$-test; $p=0.0001$ controlling for working memory capacity; $p=0.0001$ controlling for weight), resulting in a more linear mapping of objective probabilities and less heterogeneity in probability distortions (Figure $3 \mathrm{~b}$ and e). Specifically, in the amisulpride group, $\alpha$ values ranged from 0.56 to 1.52 , whereas in the placebo group they ranged from 0.26 to 1.56 (Figure 3d). Bartlett's test for the equality of variances in the revealed functions showed a significantly smaller spread in probability distortion in the amisulpride group (Bartlett's test statistic $=7.79 ; p=0.005)$. This more linear mapping of objective probabilities under D2/D3 receptor blockade could result in less risk aversion for lotteries with typically large probabilities that were underweighted by the placebo group.

To determine whether reduced utility curvature or probability distortion was the primary driver of reduced risk aversion under amisulpride, we regressed participants' choice frequency of high risk and high expected value options against treatment $\times$ parameter interactions simultaneously (multiple linear regression). For high-risk choices, both utility curvature $(\beta=0.15 ; p=0.0001)$ and probability distortion $(\beta=0.06 ; p=0.02)$ parameters impacted choice, but compared with probability distortion $\left(R^{2}=0.19\right)$, utility curvature explained significantly more variance $\left(R^{2}=0.28\right)$ in the frequency of riskier choices (linear hypothesis test on coefficients, $\mathrm{F}=21.58 ; p=0.0001)$. A similar pattern arose for high expected value choices (utility curvature $\beta=0.22$; $p=0.0001$; probability distortion $\beta=0.08 ; p=0.02$ ), with utility curvature explaining significantly more variance $\left(R^{2}=0.42\right.$ compared with $R^{2}=0.25$; linear hypothesis test on coefficients, $\mathrm{F}=17.49, p=0.0001$ ). Thus, amisulpride effects on both parameters significantly impacted choices in our data, but within the range of decisions used in our task, the effect on the utility curvature parameter appeared to have a significantly higher impact on driving decreased risk aversion.

The two groups did not differ significantly in the recovered loss aversion parameters (amisulpride $=1.412$; placebo $=$ $1.409 ; t_{91}=-0.35, p=0.73$; two-sample $t$-test; Figure $3 \mathrm{c}$ ). A two-way ANOVA with parameter values $(\alpha, \sigma$, and $\lambda)$, and group treatment as factors confirmed that there was no significant effect of the drug on participants' loss aversion parameters $(p=0.99)$ in our task, suggesting that dopamine may be more strongly involved in coding the subjective processing of probability and gain magnitude rather than loss aversion. It may be worth noting that this analysis also 

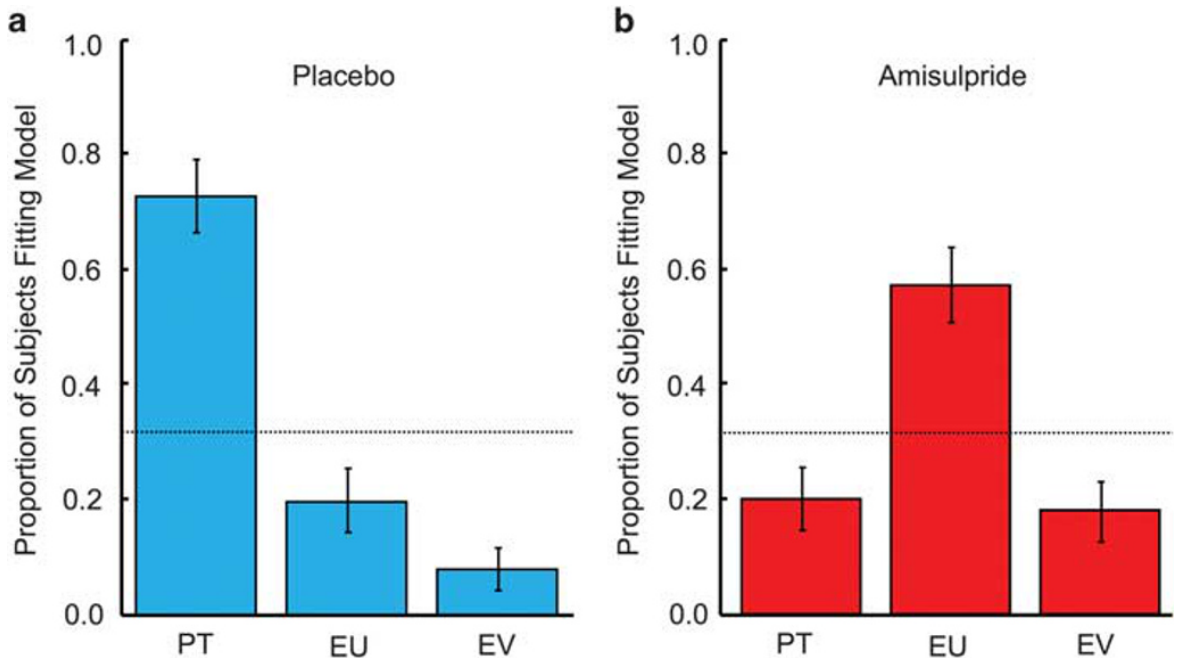

Figure 4 Estimated model frequencies from Bayesian model comparison. (a) For the placebo group, prospect theory (PT) best explained choices in 38/48 participants, and was the best fitting model overall (highest exceedance probability) in comparison with expected utility (EU) or expected value (EV) models. (b) In contrast, the expected utility model best explained choices in the amisulpride group, with 28/45 participants being classified as EU types. Dashed lines in both panels denote the probability that all models perform equally well at best fitting participants.

showed significantly less variance in the parameter estimates for the amisulpride group than the placebo group (Bartlett's test statistic $=17.62, p=0.001$; Figure $3 \mathrm{c}$ ), again indicating that amisulpride did not simply increase randomness in choice behavior. Overall, utility curvature and probability distortion were significantly correlated $\left(R^{2}=0.27 ; p<0.001\right)$. There was no significant correlation between utility curvature and loss aversion $\left(R^{2}=0.03 ; p=0.09\right)$ or probability distortion and loss aversion $\left(R^{2}=0.03 ; p=0.11\right)$. For the amisulpride group, there was no correlation in parameter values and behavioral measures with weight (all $p$-values $>0.1$ ).

Finally, we computed both the Akaike information criterion (AIC) and Bayesian information criterion (BIC) for each model fit, penalizing more complex models by correcting for the number of free parameters. We then used Bayesian model comparison to determine which model (prospect theory, expected utility, or expected value) best explained the observed choices in both groups. Focusing on AIC in the placebo group, prospect theory fitted the observed choices significantly better than expected utility and expected value models (Figure 4a; exceedance probability $=1.0$; $p=0.001 ; 38 / 48$ participants classified as prospect theory types). In striking contrast, in the amisulpride group, expected utility theory fitted behavior significantly better than prospect theory (Figure $4 \mathrm{~b}$; exceedance probability $=$ $1.0 ; p=0.03 ; 28 / 45$ participants classified as expected utility types). Comparison of BIC also confirmed this result (Supplementary Figure S4). Thus, the expected utility model best explained choice behavior when D2 receptor action was blocked.

\section{DISCUSSION}

Our results indicate a key role for the dopaminergic system in the processing of subjective values during risky decision making. Specifically, D2/D3 receptor antagonism reduced risk aversion. Computational modeling revealed that this effect arose from two channels: an increase in the linearity of the utility curvature, reflected in higher sensitivity for larger reward magnitudes, and a decrease in probability distortion. By extension, the expected utility model explained choice behavior best under amisulpride, whereas prospect theory had the highest explanatory power under placebo.

By concurrently and systematically estimating all three parameters governing risky decision making according to prospect theory, we assessed the effects of D2/D3 receptor blockade on each parameter separately, providing a clear advance to the literature (St Onge and Floresco, 2009; Kandasamy et al, 2014; Sokol-Hessner et al, 2015). The linearized utility curvature and probability distortion functions under amisulpride are exactly what would be expected if dopaminergic projections and dopaminoceptive neurons of the nigrostriatal or mesolimbic systems are involved in coding the subjective value of risky choice options. Indeed, the two parameters co-determine subjective value and affect choice, both in humans (Kahneman and Tversky, 1979) and other animals, such as non-human primates (Stauffer et al, 2015).

Previous studies have clearly demonstrated that dopaminergic manipulation has an effect on risk taking. For example, L-DOPA (which, after conversion to dopamine, stimulates both D1 and D2 receptors concurrently) has been shown to increase choices for riskier gambles involving small-stake gains (but not those involving small-stake losses) and increase baseline gambling propensity (Rutledge et al, 2015; Rigoli et al, 2016) in a value-independent manner. In line with these findings, our data show that dopamine is important for risk taking and that it is less involved for loss than for gain processing during risky choices. In addition, the present findings reveal that receptor-specific dopamine manipulations can affect risky choice and that the effects arise through effects on utility and probability distortion. Moreover, our data show that the effect of amisulpride depends on value and is particularly pronounced at high expected values. This finding is in line with a multiplicative rather than an additive effect of the drug on value processing (Zhang et al, 2009) and suggests that it may be important to 
use a large range of possible outcomes. The finding that dopamine D2/D3 receptor blockade also reduces discounting of larger later rewards (Weber et al, 2016) suggests that the mechanism of increased sensitivity to larger gain magnitudes generalizes to other forms of value-based decision making.

A major target for dopamine neurons is the striatum and other parts of the basal ganglia that regulate the selection $v s$ inhibition of actions through a direct pathway onto internal globus pallidus and substantia nigra pars reticulata $v s$ an indirect pathway on these structures via the external globus pallidus. The direct, D1-mediated (go) pathway facilitates the learning of actions to approach rewards, whereas the indirect D2-mediated (no-go) pathway inhibits behavioral responses and facilitates learning from subjectively less valued outcomes (Cox et al, 2015; Kravitz et al, 2012). Dopamine has opposing actions in the two pathways. At D2 neurons, dopamine facilitates inhibition of the indirect pathway by impeding up-state transitions, diminishing up-state spiking, and promoting long-term depression at excitatory glutamatergic synapses (Surmeier et al, 2014). Accordingly, postsynaptic D2 antagonism should boost the indirect pathway, resulting in increased competition between the direct and the indirect pathway and possibly other regions of the brain (Lee et al, 2016). In this regard, it is worth noting that dopamine actions on D4 receptors in the cingulate cortex (Cocker et al, 2016) and D2 receptors in the insular cortex (Ishii et al, 2015) affect risk taking. As the indirect pathway is thought to be associated with no-go responses, one speculative notion may be that D2 antagonism renders actions and alternatives that would normally not be chosen more viable and subjectively valuable. This notion is consistent with our finding that under amisulpride more risk-neutral choices were facilitated by both high-magnitude and high-probability choices, resulting in the selection of actions that would not normally be chosen. The notion is also consistent with our previous findings of amisulpride reducing reward impulsivity (Weber et al, 2016) and of amisulpride enhancing vs reducing generosity in men vs women (Soutschek et al, 2017).

More specifically, blocking D2 receptors may prevent the value-reducing action of D2-neuron stimulation by dopamine, providing a mechanistic explanation of why people became more risk seeking in our study. This interpretation concurs with the finding that stimulating D2 neurons in the nucleus accumbens during the decision phase decreases risky choice of risk-seeking rats in the stimulated trial (Zalocusky et al, 2016). Thus, D2-neuron stimulation by dopamine may reduce the value of risky choice options and our data suggest that this effect could be prevented by amisulpride. Related effects have also been demonstrated in learning situations, with the D2 antagonists such as haloperidol facilitating learning from rewards and D2 agonists such as cabergoline reducing the ability to learn from rewards (Frank et al, 2004, 2007). However, to fully test the possibility that D2 blockade is specifically involved in utility curvature and probability distortion and exclude a less specific effect of reduced dopamine action, it would be necessary to perform a similar experiment with D1-receptorspecific compounds.

Our results also lend weight to the role of D1 and D2 receptors in the regulation of value encoding proposed by a recent model of uncertain reward learning in the basal ganglia that postulates reward magnitudes are encoded in the difference between the synaptic weights of D1 and D2 neurons, whereas reward uncertainty is coded in the sum (Mikhael and Bogacz, 2016). Blocking the D2-mediated dopaminergic pathway could increase the difference between D1 and D2 synaptic weights, causing a concomitant increase in magnitude sensitivity as shown in our data. The sum of synaptic activity should also decrease, thus decreasing uncertainty coding and causing a decrease in risk aversion relative to the placebo group, offering an alternative potential mechanism for the reduction of risk aversion reported here.

Although amisulpride reduced risk aversion, it did not cause participants to become risk seeking, evidenced by the increased linearity of the utility and probability weighting functions. It remains unclear if higher doses of D2 antagonists would cause the shape of the utility function to become convex (and thus induce risk seeking by further increasing sensitivity to increasing monetary magnitudes). The literature on medication-induced pathological gambling in Parkinson' disease (Molina et al, 2000; Driver-Dunckley et al, 2003) may suggest that this possibility is worthy of further investigation.

Amisulpride not only increased sensitivity to reward magnitude but also reduced probability distortion. This effect is in line with the expected utility model of risky decision making (Bernoulli, 1954; Von Neumann and Morgenstern, 2007) and was reflected in the increased fit of this model compared with prospect theory under amisulpride. In contrast, under placebo, prospect theory provided a better model fit than expected utility. Together, these findings suggest that deviations from expected utilitylike decision making are driven by D2 actions.

In contrast to the effects on magnitudes and probabilities, our data showed that loss aversion remained unaffected by an acute dose of amisulpride. This finding is compatible with the notion that dopamine preferentially processes the value of rewards rather than punishments (Fiorillo, 2013). However, it has been shown that tonic stimulation of D2/D3 receptors can change the subjective value of losses (Campbell-Meiklejohn et al, 2011) and reduce negative reward prediction error encoding (van Eimeren et al, 2009; but see Pessiglione et al, 2006). It is conceivable that using higher doses of amisulpride would have affected loss aversion in our task. However, it is worth keeping in mind that the subjective definition of a loss is highly dependent on the reference point that may vary across experimental designs and participants (Walasek and Stewart, 2015).

Amisulpride is relatively selective for D2/D3 receptors but also acts on serotonergic 5-HT7 receptors (Abbas et al, 2009). Although it has been related to memory formation and sleep (Gasbarri and Pompili, 2014), the role of the 5HT7 receptor for value-based decisions is largely unknown. However, given that serotonin (5-HT) has been associated with punishment processing and response inhibition (Cools et al, 2008) and with counteracting dopamine (Daw et al, 2002), it seems unlikely that the present effects are due to 5-HT7 actions of amisulpride. Another limitation to the interpretation of our results is that the effects of dopaminergic drugs on cognitive functions are sensitive to baseline dopamine synthesis capacity that we measured only indirectly in the current study through digit span (Cools et al, 2009). The fact that all our effects were robust to 
inclusion of digit span data raises the question of whether baseline synthesis capacity plays less of a role for risky decision making than for more cognitive tasks. Although our groups were matched for BMI, another potential limitation is that we did not adjust $\mathrm{mg} / \mathrm{kg}$ dosage per participant in the amisulpride group or take blood plasma readings to assess drug uptake at the time of the task. Our previous research showed little relation between blood plasma levels of amisulpride and value-based behavior (Weber et al, 2016). Moreover, we did not find strong correlations between weight and behavioral effects. However, to fully assess the consequences of D2 antagonism on value processing it would be necessary to investigate dose response curves in future studies. Finally, the temporal specificity of D2 antagonism is difficult to judge using our study design, and the question of whether amisulpride affects decisions at the time point of valuation or choice remains to be determined.

In conclusion, this research sheds light on the specific role of dopaminergic activity in encoding subjective reward magnitude and probabilities during risky choice. Blockade of D2/D3 receptors straightened both value and probability weighting functions, resulting in more risk neutrality. Moreover, our findings specify the mechanisms that may underlie behavioral side effects of dopaminergic medicines (either antagonistic or agonistic) used in the treatment of psychiatric and neurological disorders and demonstrate the differential roles of the D1- and D2-mediated pathways in the processing of value in risky decision making.

\section{FUNDING AND DISCLOSURE}

The authors declare no conflict of interest.

\section{ACKNOWLEDGMENTS}

We are grateful to Olivier Toubia and Eric Johnson for helpful input regarding model fitting and Robert Schreiber and Linda Horvath for help with data collection. This work was supported by funding from Swiss National Science Foundation grants PDFMP1-123113/1 (to ARB), PP00P1_128574, PP00P1_150739, and 00014_165884 (to PNT), University of Zurich Forschungskredit grant FK-16-016 (to CJB), and ERC advanced grant 295642 (to EF).

\section{AUTHOR CONTRIBUTIONS}

Conceptualization: CJB, AS, SW, ARB, and PNT; methodology: CJB, AS, and PNT; formal analysis: CJB, AS, and PNT; investigation: $\mathrm{CJB}, \mathrm{AS}, \mathrm{ARB}$, and $\mathrm{HH}$; writing (original draft: CJB and PNT; writing (review and editing): CJB, AS, EF, and PNT; supervision: PNT; funding acquisition: EF and PNT.

\section{REFERENCES}

Abbas AI, Hedlund PB, Huang XP, Tran TB, Meltzer HY, Roth BL (2009). Amisulpride is a potent 5-HT7 antagonist: relevance for antidepressant actions in vivo. Psychopharmacology 205: 119-128.

Bernoulli D (1954). Exposition of a new theory on the measurement of risk. Econometrica 22: 23-36.

Bornovalova MA, Daughters SB, Hernandez GD, Richards JB, Lejuez CW (2005). Differences in impulsivity and risk-taking propensity between primary users of crack cocaine and primary users of heroin in a residential substance-use program. Exp Clin Psychopharmacol 13: 311-318.

Campbell-Meiklejohn D, Wakeley J, Herbert V, Cook J, Scollo P, Ray MK et al (2011). Serotonin and dopamine play complementary roles in gambling to recover losses. Neuropsychopharmacology 36: 402-410.

Christopoulos GI, Tobler PN, Bossaerts P, Dolan RJ, Schultz W (2009). Neural correlates of value, risk, and risk aversion contributing to decision making under risk. J Neurosci 29: 12574-12583.

Clark CA, Dagher A (2014). The role of dopamine in risk taking: a specific look at Parkinson's disease and gambling. Front Behav Neurosci 8: 196.

Cocker PJ, Hosking JG, Murch WS, Clark L, Winstanley CA (2016). Activation of dopamine D4 receptors within the anterior cingulate cortex enhances the erroneous expectation of reward on a rat slot machine task. Neuropharmacology 105: 186-195.

Cools R, Frank MJ, Gibbs SE, Miyakawa A, Jagust W, D'Esposito M (2009). Striatal dopamine predicts outcome-specific reversal learning and its sensitivity to dopaminergic drug administration. J Neurosci 29: 1538-1543.

Cools R, Roberts AC, Robbins TW (2008). Serotoninergic regulation of emotional and behavioural control processes. Trends Cogn Sci 12: $31-40$.

Cornsweet TN (1962). The staircase-method in psychophysics. Am J Psychol 75: 485-491.

Cox SM, Frank MJ, Larcher K, Fellows LK, Clark CA, Leyton M et al (2015). Striatal D1 and D2 signaling differentially predict learning from positive and negative outcomes. Neuroimage 109: 95-101.

Daw ND, Kakade S, Dayan P (2002). Opponent interactions between serotonin and dopamine. Neural Netw 15: 603-616.

Driver-Dunckley E, Samanta J, Stacy M (2003). Pathological gambling associated with dopamine agonist therapy in Parkinson's disease. Neurology 61: 422-423.

Fiorillo CD (2013). Two dimensions of value: dopamine neurons represent reward but not aversiveness. Science 341: 546-549.

Frank MJ, Samanta J, Moustafa AA, Sherman SJ (2007). Hold your horses: impulsivity, deep brain stimulation, and medication in parkinsonism. Science 318: 1309-1312.

Frank MJ, Seeberger LC, O'Reilly RC (2004). By carrot or by stick: cognitive reinforcement learning in parkinsonism. Science 306: 1940-1943.

Gasbarri A, Pompili A (2014). Serotonergic 5-HT7 receptors and cognition. Rev Neurosci 25: 311-323.

Howe MW, Tierney PL, Sandberg SG, Phillips PE, Graybiel AM (2013). Prolonged dopamine signalling in striatum signals proximity and value of distant rewards. Nature 500: 575-579.

Ishii H, Ohara S, Tobler PN, Tsutsui K, Iijima T (2015). Dopaminergic and serotonergic modulation of anterior insular and orbitofrontal cortex function in risky decision making. Neurosci Res 92: 53-61.

Kahneman D, Tversky A (1979). Prospect theory: an analysis of decision under risk. Econometrica 47: 263-291.

Kandasamy N, Hardy B, Page L, Schaffner M, Graggaber J, Powlson AS et al (2014). Cortisol shifts financial risk preferences. Proc Natl Acad Sci USA 111: 3608-3613.

Kravitz AV, Tye LD, Kreitzer AC (2012). Distinct roles for direct and indirect pathway striatal neurons in reinforcement. Nat Neurosci 15: 816-818.

Le Bricon C, Hulot T, Canal M, Hortan N, Lins RL (1996). Pharmacokinetics of amisulpride and its enantiomers after single and repeated doses in healthy volunteers. Eur J Drug Metab Pharmacokinet (special issue) 135-136.

Lee HJ, Weitz AJ, Bernal-Casas D, Duffy BA, Choy M, Kravitz AV et al (2016). Activation of direct and indirect pathway medium spiny neurons drives distinct brain-wide responses. Neuron 91: $412-424$ 
Leland DS, Paulus MP (2005). Increased risk-taking decisionmaking but not altered response to punishment in stimulantusing young adults. Drug Alcohol Depend 78: 83-90.

Meisenzahl EM, Schmitt G, Gründer G, Dresel S, Frodl T, la Fougère $\mathrm{C}$ et al (2008). Striatal D2/D3 receptor occupancy, clinical response and side effects with amisulpride: an iodine-123iodobenzamide SPET study. Pharmacopsychiatry 41: 169-175.

Mikhael JG, Bogacz R (2016). Learning reward uncertainty in the basal ganglia. PLoS Comp Biol 12: e1005062.

Molina JA, Sáinz-Artiga MJ, Fraile A, Jiménez-Jiménez FJ, Villanueva C, Ortí-Pareja M et al (2000). Pathologic gambling in Parkinson's disease: a behavioral manifestation of pharmacologic treatment? Mov Disord 15: 869-872.

Müller MJ, Wetzel H, Eich FX, Rein W, Puech A, Benkert O (2002). Dose-related effects of amisulpride on five dimensions of psychopathology in patients with acute exacerbation of schizophrenia. J Clin Psychopharmacol 22: 554-560.

Pascal B (1948). Great Shorter Works of Pascal. Greenwood Press: Westport, CT.

Pessiglione M, Seymour B, Flandin G, Dolan RJ, Frith CD (2006). Dopamine-dependent prediction errors underpin reward-seeking behaviour in humans. Nature 442: 1042-1045.

Prelec D (1998). The probability weighting function. Econometrica 66: 497-527.

Racagni G, Canonico PL, Ravizza L, Pani L, Amore M (2004). Consensus on the use of substituted benzamides in psychiatric patients. Neuropsychobiology 50: 134-143.

Rigoli F, Rutledge RB, Chew B, Ousdal OT, Dayan P, Dolan RJ (2016). Dopamine increases a value-independent gambling propensity. Neuropsychopharmacology 41: 2658-2667.

Rogers RD, Everitt BJ, Baldacchino A, Blackshaw AJ, Swainson R, Wynne K et al (1999). Dissociable deficits in the decision-making cognition of chronic amphetamine abusers, opiate abusers, patients with focal damage to prefrontal cortex, and tryptophan-depleted normal volunteers: evidence for monoaminergic mechanisms. Neuropsychopharmacology 20: 322-339.

Rosenzweig P, Canal M, Patat A, Bergougnan L, Zieleniuk I, Bianchetti G (2002). A review of the pharmacokinetics, tolerability and pharmacodynamics of amisulpride in healthy volunteers. Hum Psychopharmacol 17: 1-13.

Rutledge RB, Skandali N, Dayan P, Dolan RJ (2015). Dopaminergic modulation of decision making and subjective well-being. J Neurosci 35: 9811-9822.

Schoemaker H, Claustre Y, Fage D, Rouquier L, Chergui K, Curet O et al (1997). Neurochemical characteristics of amisulpride, an atypical dopamine D2/D3 receptor antagonist with both presynaptic and limbic selectivity. J Pharmacol Exp Ther 280: 83-97.

Schultz W (1998). Predictive reward signal of dopamine neurons. J Neurophysiol 80: 1-27.

Sokol-Hessner P, Lackovic SF, Tobe RH, Camerer CF, Leventhal BL, Phelps EA (2015). Determinants of propranolol's selective effect on loss aversion. Psychol Sci 26: 1123-1130.

Soutschek A, Burke CJ, Raja Beharelle A, Schreiber R, Weber SC, Karipidis II et al (2017). The dopaminergic reward system underpins gender differences in social preferences. Nat Hum Behav 1: 819-827.
St Onge JR, Floresco SB (2009). Dopaminergic modulation of riskbased decision making. Neuropsychopharmacology 34: 681-697.

Stauffer WR, Lak A, Bossaerts P, Schultz W (2015). Economic choices reveal probability distortion in macaque monkeys. J Neurosci 35: 3146-3154.

Stauffer WR, Lak A, Schultz W (2014). Dopamine reward prediction error responses reflect marginal utility. Curr Biol 24: 2491-2500.

Steyer R, Schwenkmezger P, Notz P, Eid M (1997). Der Mehrdimensionale Befindlichkeitsfragebogen (MDBF). Hogrefe: Göttingen.

Stopper CM, Floresco SB (2014). What's better for me? Fundamental role for lateral habenula in promoting subjective decision biases. Nat Neurosci 17: 33-35.

Stopper CM, Khayambashi S, Floresco SB (2013). Receptor-specific modulation of risk-based decision making by nucleus accumbens dopamine. Neuropsychopharmacology 38: 715-728.

Stopper CM, Maric TL, Montes DR, Wiedman CR, Floresco SB (2014). Overriding phasic dopamine signals redirects action selection during risk/reward decision making. Neuron 84: 177-189.

Sugam JA, Day JJ, Wightman RM, Carelli RM (2012). Phasic nucleus accumbens dopamine encodes risk-based decisionmaking behavior. Biol Psychiatry 71: 199-205.

Surmeier DJ, Graves SM, Shen W (2014). Dopaminergic modulation of striatal networks in health and Parkinson's disease. Curr Opin Neurobiol 29: 109-117.

Tai LH, Lee AM, Benavidez N, Bonci A, Wilbrecht L (2012). Transient stimulation of distinct subpopulations of striatal neurons mimics changes in action value. Nat Neurosci 15: 1281-1289.

Tobler PN, Fiorillo CD, Schultz W (2005). Adaptive coding of reward value by dopamine neurons. Science 307: 1642-1645.

Toubia O, Johnson E, Evgeniou T, Delquié P (2013). Dynamic experiments for estimating preferences: an adaptive method of eliciting time and risk parameters. Manage Sci 59: 613-640.

Tversky A, Kahneman D (1992). Advances in prospect theory: cumulative representation of uncertainty. J Risk Uncertainty 5: 297-323.

van Eimeren T, Ballanger B, Pellecchia G, Miyasaki JM, Lang AE, Strafella AP (2009). Dopamine agonists diminish value sensitivity of the orbitofrontal cortex: a trigger for pathological gambling in Parkinson's disease. Neuropsychopharmacology 34: 2758-2766.

Von Neumann J, Morgenstern O (2007). Theory of Games and Economic Behavior. Princeton University Press: Princeton, NJ.

Walasek L, Stewart N (2015). How to make loss aversion disappear and reverse: tests of the decision by sampling origin of loss aversion. J Exp Psychol Gen 144: 7.

Weber SC, Beck-Schimmer B, Kajdi ME, Müller D, Tobler PN, Quednow BB (2016). Dopamine D2/3- and $\mu$-opioid receptor antagonists reduce cue-induced responding and reward impulsivity in humans. Transl Psychiatry 6: e850.

Zalocusky KA, Ramakrishnan C, Lerner TN, Davidson TJ, Knutson B, Deisseroth K (2016). Nucleus accumbens D2R cells signal prior outcomes and control risky decision-making. Nature 531: 642-646.

Zhang J, Berridge KC, Tindell AJ, Smith KS, Aldridge JW (2009). A neural computational model of incentive salience. PLoS Comp Biol 5: e1000437.

Supplementary Information accompanies the paper on the Neuropsychopharmacology website (http://www.nature.com/npp) 